\title{
Impact of managed clinical networks on NHS specialist neonatal services in England: population based study
}

\author{
(@) (1) O OPEN ACCESS
}

\author{
C Gale clinical research fellow, S Santhakumaran statistician, S Nagarajan data analyst, Y Statnikov \\ data analyst, N Modi professor of neonatal medicine, on behalf of the Neonatal Data Analysis Unit \\ and the Medicines for Neonates Investigator Group
}

Section of Neonatal Medicine, Department of Medicine, Chelsea and Westminster Campus, Imperial College London, London SW10 9NH, UK

\begin{abstract}
Objective To assess the impact of reorganisation of neonatal specialist care services in England after a UK Department of Health report in 2003.

Design A population-wide observational comparison of outcomes over two epochs, before and after the establishment of managed clinical neonatal networks.

Setting Epoch one: 294 maternity and neonatal units in England, Wales, and Northern Ireland, 1 September 1998 to 31 August 2000, as reported by the Confidential Enquiry into Stillbirths and Sudden Deaths in Infancy Project 27/28. Epoch two: 146 neonatal units in England contributing data to the National Neonatal Research Database at the Neonatal Data Analysis Unit, 1 January 2009 to 31 December 2010.
\end{abstract}

Participants Babies born at a gestational age of $27^{+0}-28^{+6}$ (weeks+days): 3522 live births in epoch one; 2919 babies admitted to a neonatal unit within 28 days of birth in epoch two.

Intervention The national reorganisation of neonatal services into managed clinical networks.

Main outcome measures The proportion of babies born at hospitals providing the highest volume of neonatal specialist care ( $\geq 2000$ neonatal intensive care days annually), having an acute transfer (within the first 24 hours after birth) and/or a late transfer (between 24 hours and 28 days after birth) to another hospital, assessed by change in distribution of transfer category ("none," "acute," "late"), and babies from multiple births separated by transfer. For acute transfers in epoch two, the level of specialist neonatal care provided at the destination hospital (British Association of Perinatal Medicine criteria).

Results After reorganisation, there were increases in the proportions of babies born at 27-28 weeks' gestation in hospitals providing the highest volume of neonatal specialist care (18\% (631/3495) v49\% (1325/2724); odds ratio $4.30,95 \%$ confidence interval 3.83 to $4.82 ; \mathrm{P}<0.001)$ and in acute and late postnatal transfers (7\% (235) v12\% (360) and 18\% (579) $v 22 \%$ (640), respectively; $\mathrm{P}<0.001)$. There was no significant change in the proportion of babies from multiple births separated by transfer
(33\% (39) v 29\% (38); $0.86,0.50$ to $1.46 ; P=0.57)$. In epoch two, 32\% of acute transfers were to a neonatal unit providing either an equivalent $(n=87)$ or lower $(n=26)$ level of specialist care.

Conclusions There is evidence of some improvement in the delivery of neonatal specialist care after reorganisation. The increase in acute transfers in epoch two, in conjunction with the high proportion transferred to a neonatal unit providing an equivalent or lower level of specialist care, and the continued separation of babies from multiple births, are indicative of poor coordination between maternity and neonatal services to facilitate in utero transfer before delivery, and continuing inadequacies in capacity of intensive care cots. Historical data representing epoch one are available only in aggregate form, preventing examination of temporal trends or confounding factors. This limits the extent to which differences between epochs can be attributed to reorganisation and highlights the importance of routine, prospective data collection for evaluation of future health service reorganisations.

\section{Introduction}

The rate of preterm birth is increasing worldwide. ${ }^{1-3}$ In economically advantaged countries reported rates range from $6 \%$ to $12 \% .{ }^{12}$ In England about 10000 babies born at or below 32 weeks' gestation are admitted to National Health Service (NHS) neonatal units each year. ${ }^{4}$ The survival of preterm infants has also steadily increased over time, ${ }^{5}$ and the long term health implications are becoming increasingly apparent, ${ }^{67}$ emphasising the importance of high quality neonatal care. Delivery in specialist centres is associated with improved outcomes, ${ }^{8}$ and many countries have adopted highly regionalised systems of neonatal care. ${ }^{9}$ In the United Kingdom, neonatal services have developed largely in response to local needs. Historically most district general hospitals offered some level of specialist neonatal care, but there was considerable variation in the degree to which hospitals collaborated in the provision of intensive care..$^{10}$ 
In 2001 the Department of Health commissioned a review of neonatal services in England. Largely because of evidence that countries with network based approaches lead the way in the provision of high quality neonatal care, ${ }^{9}$ the subsequent 2003 report recommended the reorganisation of neonatal care in England into managed clinical networks, in which clusters of hospitals providing different levels of specialist care work in collaboration. The option of centralisation of services was rejected, and no specific recommendations were made with regard to volume of neonatal intensive care to be provided by the most highly specialised centres. The principal recommendations of the 2003 report were to improve the provision of high quality specialist neonatal care and the survival of babies admitted to neonatal units. Specific mention was made of the importance of transferring women at high risk of preterm labour to a specialist centre before delivery, so reducing the number of babies requiring acute postnatal transfer. Inherent to the concept of managed clinical networks is the provision of highly specialised intensive care at a limited number of centres, followed by transfer back for convalescent care to a less specialised centre closer to home. The Department of Health report made no recommendation for a formal evaluation of the impact of this major national reorganisation but noted the importance of collecting high quality data. The ensuing reorganisation was a major stimulus for the progressive introduction of electronic health record systems incorporating the capture of standard data items. These electronic data are held by an authorised NHS hosting company and released to the Neonatal Data Analysis Unit (NDAU) subject to approval from the Caldicott Guardians of each contributing NHS Trust. The unit was established in 2007 at Imperial College London as an independent academic unit to use operational NHS clinical data to support neonatal specialist services and research.

We examined the impact of the national reorganisation of neonatal services in England on key objectives reflecting the Department of Health recommendations. We tested the hypotheses that between the times before (epoch one) and after (epoch two) the establishment of managed clinical neonatal networks in England there has been an increase in the proportion of babies born at 27-28 weeks' gestation at a hospital providing the highest specialist neonatal intensive care activity, a decrease in the proportion transferred within the first 24 hours after birth (acute transfer), an increase in the proportion transferred between 24 hours and 28 days after birth (late transfer), and a reduction in the proportion of babies in twin or higher order birth sets who are separated by transfer. We also compared the rate of transfers in singleton and multiple births across epochs and measured changes in 28 day mortality.

As secondary aims, we examined the pattern of postnatal transfers in epoch two in relation to place of birth, age at transfer, and whether acute transfers were to a neonatal unit providing a higher, lower, or equivalent level of specialist care.

\section{Methods}

\section{Data sources}

Epoch one-Data for the period before the reorganisation (epoch one, 1 September 1998 to 31 August 2000) were extracted from the published report of the Confidential Enquiry into Stillbirths and Deaths in Infancy (CESDI) Project 27/28, commissioned by the Maternal and Child Health Research Consortium. The project identified the 28 day outcome of all babies born alive at 27-28 weeks' gestation in England, Wales and Northern Ireland over a two year period (1 September 1998 to 31 August 2000) and undertook related confidential enquiries to examine quality of care copies of the full CESDI report and Project 27/28 are available in appendix 1 and 2 on bmj.com). Briefly, log books were introduced to all labour wards and neonatal units in England, Wales, and Northern Ireland, with regional coordinators responsible for collection of a dataset (see appendix 2 on bmj.com) on all births within the target gestational range. All live births were included, including deaths on the labour ward before admission to a neonatal unit. Regional coordinators also provided descriptions of each hospital. This included the number of deliveries, presence of a "neonatal intensive care unit" (defined as a unit providing mechanical ventilation for $\geq 48$ hours), and the number of days of neonatal intensive care provided in 1998 defined in accordance with national criteria from the British Association of Perinatal Medicine. ${ }^{11}$ A neonatal intensive care day represents the highest category of care, involving, for example, the use of assisted ventilation, major surgery, dialysis, or cardiovascular support with inotropic agents. In the CESDI Project 27/28, hospitals were categorised on the basis of the annual number of neonatal intensive care days provided (1-499, 500-999, 1000-1499, 1500-1999, and $\geq 2000$ ). Babies were classified as "not transferred," "transferred $\leq 24$ hours," and "transferred between 24 hours and 28 days"; babies from multiple birth sets were classified as "separated due to transfer" or "not separated." No additional information on the definition of these variables was provided. Survival data were presented as number of liveborn infants surviving to 28 postnatal days. Individual patient or hospital level data were not provided. We attempted to obtain disaggregated data but were informed by the Health Care Quality Improvement Partnership, the custodians of the CESDI Project 27/28 data, that these have been destroyed. As data were available by geographical regions for "transferred $\leq 24$ hours, transferred between 24 hours and 28 days" and "survival", these outcomes were extracted for England alone. For "level of specialisation of neonatal unit at hospital of birth" and "multiple birth sets separated by transfer" data were available only for England, Wales, and Northern Ireland combined.

Epoch two-Data for epoch two were extracted from the National Neonatal Research Database held by the NDAU. These data are available at individual patient level but confined to neonatal units in England. The NDAU holds national research ethics committee approval to create a National Neonatal Research Database from these records. Data include static descriptive items captured once per baby (such as birth weight, gestational age), episodically as required (such as episodes of infection, other clinical outcomes based on unambiguous case definitions), and daily (such as treatments and procedures, level of care). Patient identifiers are removed and mother and infant NHS numbers are encrypted before data transfer to the NDAU. The National Neonatal Research Database holds information only on babies admitted to a neonatal unit and does not include details of babies who die on a labour ward before admission. One hundred and forty six of 173 neonatal units in England contributed data to the National Neonatal Research Database for the study period (epoch two, 1 January 2009 to 31 December 2010).

\section{Data extraction}

Epoch one-Aggregate data were extracted from the published report of the CESDI Project 27/28. These comprised the total number of babies born alive at 27-28 weeks' gestation by sex, plurality, birthweight category ( $\leq 500 \mathrm{~g}, 501-750 \mathrm{~g}, 751-1000$ $\mathrm{g}, 1001-1250 \mathrm{~g}, 1251-1500 \mathrm{~g}, \geq 1501 \mathrm{~g}$ ), days in neonatal intensive care (defined according to criteria from British Association of Perinatal Medicine $)^{11}$ provided by the hospital 
of birth in 1998 (categorised as none, 1-499, 500-999, 1000-1499, 1500-1999, and $\geq 2000$ ), transfer status (categorised as "not transferred," "transferred $\leq 24$ hours," and "transferred between 24 hours and 28 days"), proportion of babies from multiple births separated by transfer, and 28 day survival. Transfer category was determined by first transfer, with each baby counted only once. Data on transfer status and 28 day survival are available for England; all other data, including the number of deaths of babies of 27-28 weeks' gestation before admission to a neonatal unit, are available only as a pooled figure combining England, Wales, and Northern Ireland. The CESDI Project 27/28 report included information on a limited number of associations. Of these, details were extracted on the association between transfer status (categorised as "not transferred," "transferred $\leq 24$ hours," or "transferred between 24 hours and 28 days") with 28 day survival, and transfer status (categorised as "not transferred" or "transferred within 28 days") with multiple birth.

Epoch two-Individual patient level data on hospital of birth, gestational age, birth weight, sex, multiple birth, transfers, encrypted maternal NHS number, admission, and discharge times for each hospital episode, daily level of care, and survival for babies born at 27-28 weeks' gestation admitted to neonatal units in England for the period 1 January 2009 to 31 December 2010 were extracted from the National Neonatal Research Database; the number of babies born alive but dying before admission to a neonatal unit is not available in this database. We also obtained the annual number of neonatal intensive care days (British Association of Perinatal Medicine criteria ${ }^{11}$ ) provided by each neonatal unit, calculated as an average over the two year period. When data were not available for the entire two year period (because of late uptake of the electronic system), the equivalent annual rate was calculated based on the proportion of time for which data were available, provided that this was at least a year. Details of postnatal transfers were determined from admission and discharge times and hospital of each episode of care. Encrypted maternal NHS numbers were used to link multiple birth sets. For each multiple birth set, we compared details of admission time, discharge time, and hospital to see if the babies received care in different neonatal units simultaneously. A 12 hour window was used to allow for transfer of all babies to take place.

\section{Data analysis}

For comparisons between epochs in the proportion of babies born at 27-28 weeks' gestation at a hospital providing the highest level of specialist neonatal care activity $(\geq 2000 v<2000$ intensive care days), the proportion of multiple births separated by transfer (separated $v$ not separated), and 28 day survival, results are presented as the risk difference and odds ratio with 95\% confidence intervals. Differences between epochs one and two in population characteristics (sex, plurality, and birth weight), the distribution of annual intensive care activity by hospital of birth (none, 1-499, 500-999, 1000-1499, 1500-1999, $\geq 2000$ neonatal intensive care days), and transfer status ("not transferred," "transferred $\leq 24$ hours," "transferred between 24 hours and 28 days") were tested with $\chi^{2}$ tests. We used multivariable logistic regression to examine the association between 28 day survival and transfer, and between transfer and multiple birth, with the inclusion of interaction terms to test whether the association differed between epochs. Missing data were excluded from both $\chi^{2}$ and logistic regression analyses. When possible, we investigated the impact of missing data using sensitivity analyses. We were unable to adjust for birth weight, sex, gestational age, or disease severity in analyses of survival as these data were available only in aggregate for epoch one. All analyses were performed with SAS version 9.1.3 (SAS Institute, Cary, NC) and Stata 11 (StataCorp, College Station, TX).

\section{Sensitivity analyses}

The neonatal intensive care activity of the hospital of birth was not known for some babies in epoch two. These missing data cannot be ignored as they might be correlated with the neonatal intensive care experience of that hospital. We therefore carried out a sensitivity analysis to investigate the minimum change in odds ratio between epochs for birth at a hospital providing $\geq 2000$ versus $<2000$ intensive care days annually, assuming an extreme case for missing data. ${ }^{12}$ All babies with missing data in epoch one were assumed to have been born in hospitals providing $\geq 2000$ or more neonatal intensive care days annually; all babies with missing data in epoch two were assumed to have been born in hospitals providing $<2000$ neonatal intensive care days annually. For this outcome, data in epoch one were available only for England, Wales, and Northern Ireland combined, while epoch two data were available only for England. To investigate the potential effect of this discrepancy, we carried out a sensitivity analysis assuming that all births in Wales and Ireland occurred at hospitals providing $<2000$ intensive care days. In addition, we also examined the combined effect of both sensitivity analysis assumptions. Sensitivity analyses were also performed to investigate the effect of missing 28 day survival data, and the inclusion of deaths before admission to a neonatal unit in epoch one, on the estimated change in survival between epochs.

\section{Clustering effects}

Data from both epochs could be clustered because of similarities between babies within hospitals, countries (for epoch one), and neonatal networks (for epoch two). As only aggregate data were available for epoch one, we cannot fully account for this clustering. To investigate variation from clustering in epoch two, however, we calculated the outcome for each network and presented the median and range of these network specific outcomes. We used partially nested random effects models ${ }^{13}$ to estimate the change between epochs averaged over all neonatal networks. These models allow for clustering to be present in only part of the data. The model is of the form:

$\operatorname{logit}\left(\mathrm{p}_{\mathrm{i}}\right)=\propto+\beta \operatorname{epoch}(\mathrm{i})+\mathrm{u}_{\text {network }(i)}$ epoch $(\mathrm{i})$

where epoch(i) $=0$ for epoch one and 1 for epoch two, and network(i) is given dummy values for epoch one. The average effect across all clusters is given by $\beta$. The u's are the random effects, which are only estimated for epoch two, assumed to be normally distributed with mean 0 and variance $\sigma_{u}{ }^{2}$ estimated from the data. The intercept, $\propto$, is fixed rather than random as there is no clustering in epoch one. The network average odds ratio $(\exp \beta)$ and $95 \%$ confidence intervals are presented, together with the $\mathrm{P}$ value testing the null hypothesis that the variance between networks is equal to zero, from the likelihood ratio test. This was performed for all binary outcomes except separation because of transfer as the number of babies per network for this outcome was small.

\section{Categorisation of neonatal units}

In the CESDI Project 27/28, neonatal units were categorised on the basis of the annual number of neonatal intensive care days provided. This is not consistent with contemporary UK practice or the international literature. Therefore to provide some measure of the validity of the approach we determined the 
agreement with categories of neonatal unit according to the British Association of Perinatal Medicine: level 1 (provides special care but does not aim to provide any continuing high dependency or intensive care), level 2 (provides high dependency care and some short term intensive care), and level 3 (provides the whole range of medical neonatal care). ${ }^{14}$ To facilitate international comparison we also provide the average annual number of very low birthweight babies $(<1500 \mathrm{~g})$ admitted to each neonatal unit over the study period.

\section{Results}

During epoch one, the CESDI Project 27/28 study identified 294 centres providing maternity services in England, Wales, and Northern Ireland and 148 "neonatal intensive care units" (defined as providing mechanical ventilation for a period of more than 48 hours) (see appendix 1 on bmj.com. Seventy seven of the neonatal intensive care units received referrals, and 71 cared for babies born only within the same hospital. Of the centres providing maternity services, neonatal specialist care was not provided by 110 (of which 51 were midwife led and eight were GP led) and was offered on a limited basis by 36 (see appendix 2 on bmj.com). There were 3522 babies born alive at a gestational age from $27^{+0}$ weeks to $28^{+6}$ weeks; of these, 31 babies died before admission to a neonatal unit.

For epoch two, data were available for 146 (34 level 1, 72 level 2, 40 level 3) of 173 neonatal units in the 23 managed clinical networks in England. There were 2919 babies born at a gestational age from $27^{+0}$ to $28^{+6}$ weeks and admitted to a neonatal unit. There were significant but clinically small differences between epochs in the distribution of babies by plurality and birth weight. Table 1 shows population characteristics by epoch $\downarrow$.

\section{Primary outcomes}

The proportion of babies delivered at hospitals with the highest specialist care activity ( $\geq 2000$ neonatal intensive care days a year) increased significantly, from 18\% (631/3495) in England, Wales, and Northern Ireland in epoch one to 49\% (1325/2724) in England in epoch two (risk difference 31\%, 95\% confidence interval 28 to 33 ; odds ratio $4.30,3.83$ to 4.82 ; $\mathrm{P}<0.001$ ). Examination of the distribution of annual intensive care activity by hospital of birth (table $2 \Downarrow$ ) showed that in epoch two a smaller proportion of babies was delivered in centres across all categories representing $<2000$ neonatal intensive care days annually $\left(\mathrm{P}<0.001 ; \chi^{2}\right.$ test). The neonatal intensive care activity of hospital of birth was not known for 27 babies in epoch one and 195 babies in epoch two. A sensitivity analysis assuming babies with missing data in epoch one were born in hospitals providing $\geq 2000$ neonatal intensive care days annually, and assuming babies with missing data in epoch two were born in hospitals providing $<2000$ neonatal intensive care days annually, yielded a risk difference of $27 \%$ (24 to $29 \%$; odds ratio 3.62, 3.24 to $4.05 ; \mathrm{P}<0.001)$. A sensitivity analysis omitting births in Wales and Northern Ireland, assuming these occurred at hospitals providing <2000 neonatal intensive care days annually, resulted in a risk difference of $29 \%$ (27 to $31 \%$; odds ratio 3.89 , 3.47 to 4.36 ). Combining both these assumptions reduced the risk difference of $25 \%$ (23 to 27\%; odds ratio 3.27, 2.92 to 3.66).

Across neonatal networks the median of the proportions of babies delivered at hospitals with the highest specialist care activity in epoch two was $44.8 \%$ (range $0-79.5 \%$ ). The analysis accounting for clustering in epoch two resulted in a network averaged odds ratio of 3.25 (2.02 to 5.03), with significant variation between networks $(\mathrm{P}<0.001)$.
The proportions of babies undergoing acute and late postnatal transfer in England increased significantly between epochs, from $7 \%(235 / 3248)$ to $12 \%(360 / 2919)$ and from $18 \%$ $(579 / 3248)$ to $22 \%(640 / 2919)$, respectively $\left(\chi^{2} \mathrm{P}<0.001\right)$ (table $3 \Downarrow)$.

During epoch one, in England, Wales, and Northern Ireland, there was no significant difference in the proportion of babies from multiple births and singleton births who underwent transfer before 28 days (multiple 26\% (220/847) $v$ singleton $24 \%$ (634/2675); odds ratio $1.13,0.95$ to $1.35 ; \mathrm{P}=0.18)$. In epoch two, in England, 37\% (291/785) of multiples and 33\% (709/2134) of singletons were transferred $(1.18,0.99$ to 1.40 ; $\mathrm{P}=0.05)$. The difference in odds ratios between epochs was not significant ( $\mathrm{P}=0.71$ from interaction test).

In epoch two maternal data required to link siblings was available for $70 \%$ (549/785) of multiple births, comprising 310 sets of twins/triplets. There was no significant difference detected between epochs in the proportion of transferred twins/triplets of 27-28 weeks' gestation who were separated (epoch one, England, Wales, and Northern Ireland: 33\% (39/119); epoch two, England: 29\% (38/129)), although the small numbers limit power to detect a difference. The difference between epochs was not significant (risk difference for epoch two $v$ epoch one $-3 \%,-15$ to 8 ; odds ratio $0.86,0.50$ to 1.46 ; $\mathrm{P}=0.57$ ). A sensitivity analysis was not performed for this outcome as we could not determine the number of birth sets for babies with missing maternal data.

\section{Survival}

Survival was significantly higher in England in epoch two compared with epoch one: $94 \% v 88 \%$, risk difference $5.6 \%$ (4.2 to 7.0); odds ratio 2.00 (1.67 to 2.40 ); $\mathrm{P}<0.001$ ) (table $4 \Downarrow$ ). This difference remained significant after multivariable logistic regression adjustment for transfer status (odds ratio 1.93, 1.61 to $2.32 ; \mathrm{P}<0.001)$. The association between transfer status and survival did not differ between epochs $(\mathrm{P}=0.15$ from likelihood ratio test comparing models with and without interaction between transfer and survival). Across neonatal networks the median (range) of proportions of babies surviving in epoch two was $93 \%$ (90\% to 97\%). The analysis accounting for clustering in epoch two showed no evidence of variation across networks ( $\mathrm{P}>0.9)$. The network averaged odds ratio was 1.99 (1.58 to 2.81) after adjustment for transfer.

Data on survival at 28 days were missing for $2 \%$ (60/2919) of babies in epoch two. We investigated the impact of missing data on the odds ratio with sensitivity analyses, assuming both a best and worst case scenario. For the best case scenario we assumed that all babies with missing outcomes survived to 28 days, yielding an adjusted odds ratio of 1.96 (1.63 to 2.35 ; $\mathrm{P}<0.001$ ). For the worst case scenario we assumed that all babies with missing outcomes did not survive to 28 days, yielding an adjusted odds ratio of 1.93 (1.61 to $2.32 ; \mathrm{P}<0.001$ ).

Thirty one babies in epoch one died before admission to a neonatal unit. This number related to England, Wales, and Northern Ireland. As the number for England alone was not available, we were unable to obtain a figure comparable with epoch two, when data related only to babies admitted to a neonatal unit. We performed a sensitivity analysis based on the extreme case assumption that all deaths before neonatal unit admission occurred in England. Exclusion of the 31 deaths before neonatal unit admission in epoch one resulted in a slight increase in survival from $88 \%$ to $89 \%$ in epoch one and a change in the odds of survival in epoch two compared with epoch one 
from 2.00 (1.67 to $2.40 ; \mathrm{P}<0.001)$ to 1.84 (1.53 to 2.22; $\mathrm{P}<0.001)$.

\section{Secondary outcomes}

There were $351 / 360$ (98\%) transfers within the first 24 hours after birth in epoch two, when information on both hospital of birth and destination neonatal unit were available. Of these $68 \%$ (238/351) were to a neonatal unit providing a higher, $25 \%$ (87/351) an equivalent, and 7\% (26/351) a lower level of specialist care. $^{14}$

Table $5 \Downarrow$ shows data on the transfer of babies in epoch two in relation to the neonatal intensive care activity of the hospital of birth. Of babies born at 27-28 weeks' gestation delivered at hospitals providing the lowest volume of neonatal intensive care (<500 intensive care days a year), 46\% (153/331) were transferred within 24 hours of birth and $42 \%$ (142/331) were never transferred, receiving all their care in the hospital of birth. Of babies delivered at hospitals with the highest neonatal intensive care activity ( $\geq 2000$ intensive care days a year), $7 \%$ (88/1325) were transferred to another neonatal unit within 24 hours of birth.

Table $6 \Downarrow$ shows the relation between neonatal unit designation ${ }^{14}$ (level 1, level 2, level 3) and volume of activity (annual number of neonatal intensive care days) in epoch two. Of 40 neonatal units designated as level 3, 29 provided $\geq 2000$ neonatal intensive care days annually in 2009-10. Table 6 also shows the relation between neonatal unit designation ${ }^{14}$ in epoch two and the number of very low birthweight babies admitted annually in 2009-10. All 11 neonatal units that provided care for 100 or more very low birthweight babies annually have level 3 designation. Table $7 \Downarrow$ shows the number of neonatal intensive care days in epoch two in relation to the number of admissions of very low birthweight babies. The 32 neonatal units providing $\geq 2000$ neonatal intensive care days annually all admitted $\geq 50$ very low birthweight babies. Re-examination of the primary outcome- the proportion of babies born at 27-28 weeks' gestation delivered "at a hospital providing the highest specialist care activity" using British Association of Perinatal Medicine designation ${ }^{14}$ — showed that 53\% (1514/2848) were delivered in a hospital with a level 3 neonatal unit (compared with $49 \%$ when we used the categorisation "hospital providing $\geq 2000$ neonatal intensive care days per annum"), 40\% (1132/2848) in a level 2 neonatal unit, and 7\% (202/2848) in a level 1 neonatal unit.

\section{Discussion}

\section{Principal findings}

Neonatal services in England were reorganised after 2003 into managed clinical networks with the aim of providing specialist care through a collaborative approach across NHS Trusts. After reorganisation there has been a significant increase (from $18 \%$ to $49 \%$ ) in the proportion of babies born 27-28 weeks' gestation in hospitals providing the highest volume of neonatal intensive care. When considered in conjunction with an increase from $18 \%$ to $22 \%$ in late transfers between 1 and 28 days, this suggests that two key aims of reorganisation are being increasingly realised-namely, to ensure babies with complex care needs are born in specialist neonatal intensive care centres and are then transferred back when clinically appropriate to a neonatal unit close to home. As the prognosis for very preterm babies is improved when they are born and have their initial care in highly specialist centres, ${ }^{8}$ the change in place of delivery we document represents an important improvement.
Our other findings, however, are less encouraging. There has been an increase from $7 \%$ to $12 \%$ in the proportion of babies born at 27-28 weeks' gestation who have an acute transfer to another hospital in the first 24 hours after birth. Some of the increase might reflect improved ability after reorganisation to effect appropriate transfer when circumstances such as the precipitate, unplanned, and unexpected onset of preterm labour results in delivery at a hospital unable to provide specialist neonatal intensive care. In this case emergency transfer to a hospital providing more specialist care is both justified and desirable. As there is a well recognised association between the emergency postnatal transfer of a preterm baby and increased mortality and morbidity, ${ }^{15-17}$ however, the intention is always to transfer the mother before delivery (in utero transfer), if clinical condition permits, so that the baby is born in an appropriately equipped hospital with staff experienced in newborn resuscitation, stabilisation, and specialist procedures. ${ }^{18}$ As over half of all babies born at 27-28 weeks' gestation are currently not delivered at a centre providing the highest volume of neonatal intensive care activity, these figures suggest that the application of a cardinal principle of high quality perinatal care highlighted by the National Audit Office ${ }^{19}$ and the National Institute for Health and Clinical Excellence ${ }^{20}$-namely, coordination between maternity and neonatal services to facilitate in utero transfer before delivery-remains poor. The proportion of babies born at hospitals with the highest neonatal intensive care activity shows significant variation across networks in epoch two, although the strength of this conclusion is limited as not all neonatal units in England contributed data and complete network coverage was not achieved. Our findings underline the limitations inherent in a major reorganisation restricted to one aspect of service provision rather than to the entire pathway of perinatal care. We also show that that almost a third of the acute transfers in epoch two were either to a neonatal unit providing an equivalent or lesser degree of expertise. Together with the continued separation of a third of multiple birth sets, this indicates continuing inadequacy in neonatal intensive care cot capacity, in keeping with evidence previously gathered by the national UK charity, Bliss. ${ }^{21}$

\section{Strengths and weaknesses}

Our analysis has several limitations. The differences between epochs might not be entirely attributable to reorganisation. In particular, we advise caution in relation to improved survival. Preterm survival has increased year on year in England and other developed countries. As only a single source of aggregated survival data was available before reorganisation we were unable to undertake trend analysis to distinguish change as a result of the reorganisation from any underlying temporal trend. For this reason we chose to focus on important organisational rather than clinical outcomes. The potential influence of confounding factors in explaining the differences in these organisational outcomes must be considered. The similar clinical characteristics of the newborn cohorts in the two epochs suggest that it is unlikely that a change in case mix accounts for the differences identified. The aggregate nature of the data for epoch one preclude comparison of outcomes adjusted for case mix. Changes in clinical practice between epochs, in particular improved prediction of preterm birth through greater use of fetal fibronectin and assessment of cervical length, ${ }^{22}$ could have altered the pattern of delivery, resulting in the increased delivery of preterm babies in neonatal units providing a higher level of care in epoch two, independent of the effect of reorganisation. This, however, would result in overestimation of the association between reorganisation and increased delivery in more 
specialised centres. During epoch one, neonatal transport services were patchy and organised on a hospital rather than a regional basis. Hence urgent acute transfers within 24 hours of birth could have been delayed more commonly in epoch one than in epoch two, an effect that again would lead to overestimation of the impact of reorganisation. For the comparisons between epochs on "delivery at hospitals with the highest intensive care activity" and "multiple birth sets separated by transfer" the historical nature of data for epoch one has meant that we have compared data from England, Wales, and Northern Ireland in epoch one with data from England alone in epoch two. In epoch one, at 27-28 weeks' gestation, births in England account for most of the births in England, Wales, and Northern Ireland combined (3248/3522 births; 92\%) (see appendix 2 on bmj.com). Therefore we consider that epoch one data representing England, Wales, and Northern Ireland is nonetheless suitably representative of practice in England before reorganisation to allow valid comparison with England only data in epoch two. To explore the possibility that the pattern of delivery differed by country in epoch one, however, we carried out a sensitivity analysis for the outcome "delivery at hospitals with the highest neonatal intensive care activity." This reduced the magnitude and precision of the odds ratio but did not alter the conclusions. To explore the difficulty presented by the lack of uniform categorisation of neonatal units during epoch one, we compared neonatal units by intensive care activity, using consistent criteria ${ }^{17}$ across both epochs. This approach is also justified by evidence that categorisation of neonatal units by intensive care activity correlates more closely with mortality than unit designation. ${ }^{23}$ As missing data could induce bias, we conducted sensitivity analyses when possible, and these did not alter our conclusions. A further difficulty resulting from the limited data available in epoch one was that we could not fully account for clustering by hospital or area. A partially nested model to account for clustering by neonatal network in epoch two reduced the magnitude and precision of the odds ratio but did not alter the conclusions. Finally, although we limited our study to babies born at 27-28 weeks' gestation to allow comparison with epoch one, this group comprises the majority of very preterm babies,${ }^{24}$ hence we suggest our conclusions are generalisable to the wider population of babies requiring specialist care.

\section{Results in the context of other studies}

Centralisation of neonatal intensive care within a smaller number of neonatal units providing both a high level of intensive care and high volume of activity is associated with reduced mortality. ${ }^{8}$ Sparsely populated countries such as Australia and Canada have shown that it is possible to achieve centralisation of specialist services across large geographical areas. ${ }^{25} 26$

Reorganisation involving maternity and newborn services has been reported from Australia, ${ }^{26}$ France,${ }^{27}$ and the United States. ${ }^{28}$ These involved regional rather than national reorganisation and smaller numbers of patients, and the impact on neonatal transfers and other aspects of care was not evaluated. Nonetheless, in each case centralisation of services seems to have resulted in upwards of $80 \%$ of very low birthweight babies being delivered in specialist neonatal intensive care centres, highlighting the extent to which services in England have yet to improve. An important question that remains to be resolved is what volume of intensive care activity leads to optimum outcomes for the baby, balancing the desirability of centralised expertise and high volume against the risks inherent in the transfer of a sick neonate. It has been suggested that the admission of at least 100 very low birthweight babies annually should be considered an appropriate target for centralisation as this reflects the volume of activity associated with improved survival. ${ }^{29}$ In 2009-10, neonatal units in England that provided 2000 or more neonatal intensive care days annually also cared for $\geq 50$ very low birthweight babies. The routine capture of standardised data by clinical teams offers opportunity for the evaluation of the impact of policy on healthcare and outcomes in patients. Future analysis of longitudinal population based data held in the National Neonatal Research Database should help to clarify this issue.

\section{Implications for clinicians and policymakers}

Managed clinical networks, defined as "linked groups of health professionals and organisations from primary, secondary and tertiary care working in a co-ordinated manner unconstrained by existing professional and [organisational] boundaries to ensure the equitable provision of high quality clinically effective services, ${ }^{30}$ have been extolled as a means to reduce costs and improve patient outcomes, though objective data justifying these conclusions are hard to find. Network based approaches vary in the extent to which coordination of services are mandated and driven by shared management, administration, and governance or represent a voluntary commitment to cooperation. ${ }^{31}$ Though complex, the former approach is considered particularly likely to improve patient outcomes where the delivery of healthcare crosses organisational, professional, and commissioning boundaries. ${ }^{32}{ }^{33}$ Central funding can provide stimulus for the development of such patient pathway based approaches to the delivery of healthcare as has been the case with cancer services and diabetes care in Scotland. ${ }^{34}$ Our study represents a useful addition to this limited literature, as to our best knowledge the impact of a national reorganisation of specialist neonatal services into managed clinical networks has not previously been examined. Our study is also relevant to other countries considering how best to structure neonatal services and topical in view of proposed changes to the NHS that could affect care pathways involving multiple providers.

We thank the staff from neonatal units involved in capturing electronic data and Richard Colquhoun, Neonatal Data Analysis Unit manager, and Surbhi Shah for administrative support.

Contributing neonatal units and clinical leads

Airedale Hospital; Andrew Gallagher, Alexandra Hospital; Donal Manning, Arrowe Park Hospital; Tim Wickham, Barnet Hospital; M Saeed, Barnsley District General Hospital; Ruth Wigfield, Basingstoke and North Hampshire Hospital; N Brookes, Bassetlaw District General Hospital; Raghavan Kadalraja, Bedford Hospital; Mike Watkinson, Birmingham Heartlands Hospital; Imogen Morgan, Birmingham Women's Hospital; S Oddie, Bradford Royal Infirmary; Manesh Babu, Broomfield Hospital; Meera Lama, Burnley General Hospital; E Crosbie, Calderdale Royal Hospital; Tim Wickham, Chase Farm Hospital; Gary Hartnoll, Chelsea and Westminster Hospital; Cheltenham General Hospital; Julie Nycyk, City Hospital; Sarah Dalton, Colchester General Hospital; Graham Whincup, Conquest Hospital; Stephen Brearey, Countess of Chester Hospital; John Chang, Croydon University Hospital; Abdul Hasib, Darent Valley Hospital; S Subramania Krishnamoorthy, Darlington Memorial Hospital; Julian Lawson, Derriford Hospital; P Adiotomre, Diana Princess of Wales Hospital; Nigel Brookes, Doncaster Royal Infirmary; Phil Wylie, Dorset County Hospital; Vivien Chan, Ealing Hospital; K Abdul Khader, East Surrey Hospital; Graham Whincup, Eastbourne District General Hospital; Ruth Shepard, Epsom General Hospital; Fairfield General Hospital; Abdus Mallik, Frimley Park Hospital; Owen Galt, Furness General Hospital; J Holman, Gloucester Royal Hospital; Mike Watkinson, Good Hope Hospital; Stanley Zengeya, Great Western Hospital; Morag Campbell, Guy's and St Thomas' Hospital; Andy Butterfill, Hereford County Hospital; Hilary Dixon, Hinchingbrooke 


\section{What is already known on this topic}

The delivery and initial management of preterm babies in hospitals appropriately staffed and equipped and experienced in the provision of neonatal specialist care is associated with improved outcomes but requires close coordination between maternity and neonatal services

The acute postnatal transfer of a sick baby for intensive care is associated with adverse outcomes

Neonatal specialist care in England was reorganised into managed clinical networks in 2003 after concerns about quality of care and outcomes. Two key aims of the reorganisation were to increase preterm deliveries at specialist centres and reduce acute postnatal transfers

\section{What this study adds}

The reorganisation of neonatal services in England into managed clinical networks has been associated with limited success in achieving stated aims

Though the proportion of babies born at 27-28 weeks' gestation in specialist centres has risen, around half continue to be delivered in non-specialist centres, indicating poor coordination between maternity and neonatal services

The proportion of preterm babies transferred within the first 24 hours has increased; in almost a third of cases this is to a neonatal unit providing an equivalent or lower level of specialist care, and babies from multiple births continue to be separated, indicating continuing inadequacies in cot capacity

Hospital; Prof Kate Costeloe, Homerton Hospital; Justin Sims, Horton Hospital; C M Wood, Hull Royal Infirmary; M James, Ipswich Hospital; Vasantha Jayalal, James Paget Hospital; Eleri Adams, John Radcliffe Hospital; B K Sharma, King George Hospital; Prof Anne Greenough, King's College Hospital; Vibert Noble, King's Mill Hospital; Dwight Lindo, Kingston Hospital; M Lama, Lancashire Women and Newborn Centre, Burnley; A Thirumurugan, Leighton Hospital; A Raffles, Lister Hospital; C W Yoxall, Liverpool Women's Hospital; P Sivakumar, Luton and Dunstable Hospital; Ignatius Losa, Macclesfield District General Hospital; Hamudi Kisat, Maidstone General Hospital; B Mohamad, Manor Hospital; Aung Soe, Medway Maritime Hospital; Jasper Katumba, Milton Keynes General Hospital; Vimala Gopinathan, Newham General Hospital; Mark Dyke, Norfolk and Norwich University Hospital; Yonas Cherinet, North Devon District Hospital; North Manchester General Hospital; Lesley Alsford, North Middlesex University Hospital; Paul Mannix, Northwick Park Hospital; Stephen Wardle, Nottingham City Hospital; Stephen Wardle, Nottingham University Hospital (QMC); Tim McBride, Ormskirk District General Hospital; Seif Babiker, Peterborough City Hospital; Minesh Khashu, Poole General Hospital; Than Soe, Princess Alexandra Hospital; Mike Hall, Princess Anne Hospital; Philip Amess, Princess Royal Hospital; Shaun Walter, Princess Royal University Hospital; C Groves, Queen Alexandra Hospital; Dennis Bosman, Queen Elizabeth Hospital, Gateshead; Susan Rubin, Queen Elizabeth Hospital, King's Lynn; Shaun Walter, Queen Elizabeth Hospital, Woolwich; Queen Elizabeth II Hospital; Mohsin Malik, Queen Elizabeth the Queen Mother Hospital; Shaun Walter, Queen Mary's Hospital, Sidcup; VK Sharma, Queen's Hospital, Romford; Rochdale Infirmary; A Ogilvy-Stuart, Rosie Maternity Hospital, Cambridge; C J Harrison, Rotherham Distric General Hospital; Vibha Sharma, Royal Albert Edward Infirmary; Greg Boden, Royal Berkshire Hospital; Royal Bolton Hospital; Paul Munyard, Royal Cornwall Hospital; John Mclntyre, Royal Derby Hospital; Vaughan Lewis, Royal Devon and Exeter Hospital; Schapira, Royal Hampshire County Hospital; Owen Galt, Royal Lancaster Infirmary; Royal Oldham Hospital; Richa Gupta, Royal Preston Hospital; Charles Godden, Royal Surrey County Hospital; Philip Amess, Royal Sussex County Hospital; Alan Fenton, Royal Victoria Infirmary; A Mohite, Russells Hall Hospital; Jonathon Moise, Salford Royal Hospital; Shirley Kinsey, Salisbury District Hospital; Julie Nycyc, Sandwell General Hospital; M Qunibi, Salisbury District Hospital; Arfa Khan, Southend Hospital; Sandra Calvert, St George's Hospital; Ruth Shephard, St Helier Hospital; S Butterworth, St Mary's Hospital, Isle of Wight; Anthony Emmerson, St Mary's Hospital, Manchester; J Tooley, St Michael's Hospital; Peter Reynolds, St Peter's Hospital; Anil Garg, St Richard's Hospital; K K Tewary, Staffordshire General Hospital; Carrie Heal, Stepping Hill Hospital; Gail Whitehead, Stoke Mandeville Hospital; Majd Abu-Harb, Sunderland Royal Hospital; Jacqueline Birch, Tameside General Hospital; Rebecca Mann, Taunton and Somerset Hospital; E Pilling, The Jessop Wing, Sheffield; M Hird, The Royal London Hospital; Stefan Schulze, Torbay Hospital; Hamudi
Kisat, Tunbridge Wells Hospital; Mark Sellwood, University College Hospital; K Blake, University Hospitals Coventry; Jauro Kuna, University Hospital Lewisham; S Subramania Krishnamoorthy, University Hospital of North Durham; K Palmer, University Hospital of North Staffordshire; Ian Verber, University Hospital of North Tees; Faisal Al-Zidgali, University Hospital of South Manchester; Ned Rowlands, Victoria Hospital, Blackpool; Ms Joan Oliver, Wansbeck General Hospital; Delyth Webb, Warrington Hospital; CA Ramesh, Watford General Hospital; Hashir Ariff, West Middlesex University Hospital; R Sanghavi, Wexham Park Hospital; Caroline Sullivan, Whipps Cross University Hospital; L Amegavie, Whiston Hospital; E Broadhurst, Whittington Hospital; David Long, William Harvey Hospital; Andrew Gallagher, Worcestershire Royal Hospital; Anil Garg, Worthing Hospital; G Whitehead, Wycombe Hospital; Megan Eaton, Yeovil District Hospital

Members of the Neonatal Data Analysis Unit and Medicines for Neonates Investigator Group

N Modi (lead), J Abbott, D Ashby, P Brocklehurst, K Costeloe, E Draper, A Majeed, J Kemp, A Young, S Petrou, A Wilkinson. The Medicines for Neonates Steering Board is chaired by Michael Goldacre.

Contributors: CG conceived the study; CG, SS, SN, YS, and NM contributed to defining the study; SN and $Y S$ prepared data for analysis by SS; CG wrote the first draft of the paper; this and subsequent drafts were revised by NM; all study authors reviewed the paper, suggested revisions, and approved the final version submitted; NM is guarantor. Funding: The Neonatal Data Analysis Unit is supported by a Programme Grant for Applied Research (RP-PG-0707-10010) from the National Institute of Health Research (NIHR) and unrestricted funding from the Department of Health, Danone, and Abbott International. The study funders had no role in study design, collection, analysis, and interpretation of data, writing of the report, or in the decision to submit the article for publication

Competing interests: All authors have completed the ICMJE uniform disclosure form at www.icmje.org/coi_disclosure.pdf (available on request from the corresponding author) and declare financial support for the submitted work from the National Institute for Health Research (NIHR) through a Programme Grant for Applied Research (RP-PG-0707-10010). SN receives part funding from the Royal College of Paediatrics and Child Health (RCPCH) National Neonatal Audit Programme. CG receives part funding though an unrelated award to NM from the Child Growth Foundation (CGF) and support from the Westminster Medical School Research Trust. SS and YS are funded by the National Institute for Health Research. The views expressed in this publication are those of the authors and not necessarily those of the RCPCH, CGF, Westminster Medical School Research Trust, NHS, NIHR, or Department of Health.

Ethical approval: This study was carried out with the National Neonatal Research Database (REC 10/H0803/151) as part of the NDAU National 
Neonatal Surveillance Programme undertaken with permission from NHS Trust Caldicott Guardian.

Data sharing: Permission is held to share data for purposes approved by a National Research Ethics Committee.

1 Norman JE, Morris C, Chalmers J. The effect of changing patterns of obstetric care in Scotland (1980-2004) on rates of preterm birth and its neonatal consequences: perinatal database study. PLoS Med 2009;6:e1000153.

2 March of Dimes. PeriStats. 2012. www.marchofdimes.com/peristats.

3 Langhoff-Roos J, Kesmodel U, Jacobsson B, Rasmussen S, Vogel I. Spontaneous preterm delivery in primiparous women at low risk in Denmark: population based study. BMJ 2006;332:937-9.

4 National Neonatal Audit Programme (NNAP). Annual report. Royal College of Paediatrics and Child Health Science and Research Department, 2009.

5 Saigal S, Doyle LW. An overview of mortality and sequelae of preterm birth from infancy to adulthood. Lancet 2008;371:261-9.

6 Crump C, Sundquist K, Sundquist J, Winkleby MA. Gestational age at birth and mortality in young adulthood. JAMA 2011;306:1233-40.

7 Thomas EL, Parkinson JR, Hyde MJ, Yap IK, Holmes E, Dore CJ, et al. Aberrant adiposity and ectopic lipid deposition characterise the adult phenotype of the preterm infant. Pediatr Res 2011;70:507-12.

8 Lasswell SM, Barfield WD, Rochat RW, Blackmon L. Perinatal regionalization for very low-birth-weight and very preterm infants: a meta-analysis. JAMA 2010;304:992-1000.

9 Hallsworth M, Ferrands A, Oortwijn WJ, Hatziandreu E. The provision of neonatal services, data for international comparisons. 2007. www.nao.org.uk/idoc. ashx?docld=5a5f4c156d2a-4ecd-a2bf-84016589e881\&version=-1.

10 Marlow N, Bryan Gill A. Establishing neonatal networks: the reality. Arch Dis Child Fetal Neonatal Ed 2007;92:F137-42.

11 British Association of Perinatal Medicine and Neonatal Nurses Association. Report of working group of the British Association of Perinatal Medicine and Neonatal Nurses Association on categories of babies requiring neonatal care. Arch Dis Child 1992;67:868-9.

12 Sterne JA, White IR, Carlin JB, Spratt M, Royston P, Kenward MG, et al. Multiple imputation for missing data in epidemiological and clinical research: potential and pitfalls. $B M$ 2009;338:b2393.

13 Roberts C, Roberts SA. Design and analysis of clinical trials with clustering effects due to treatment. Clin Trials 2005;2:152-62.

14 BAPM. Standards for hospitals providing neonatal intensive and high dependency care. 2nd ed. British Association of Perinatal Medicine, 2001.

15 Towers CV, Bonebrake R, Padilla G, Rumney P. The effect of transport on the rate of severe intraventricular hemorrhage in very low birth weight infants. Obstet Gynecol 2000;95:291-5

16 Shlossman PA, Manley JS, Sciscione AC, Colmorgen GH. An analysis of neonatal morbidity and mortality in maternal (in utero) and neonatal transports at 24-34 weeks gestation. Am J Perinatol 1997;14:449-56.

17 Mohamed MA, Aly H. Transport of premature infants is associated with increased risk for intraventricular haemorrhage. Arch Dis Child Fetal Neonatal Ed 2010;95:F403-7.

18 Kollee LA, Brand R, Schreuder AM, Ens-Dokkum MH, Veen S, Verloove-Vanhorick SP. Five-year outcome of preterm and very low birth weight infants: a comparison between maternal and neonatal transport. Obstet Gynecol 1992;80:635-8.

19 National Audit Office. Caring for vulnerable babies: the reorganisation of neonatal services in England. Stationery Office, 2007. www.nao.org.uk/idoc. ashx?docld=90ccd3cd-421f$469 \mathrm{e}-98 \mathrm{c} 6-\mathrm{e} 79 \mathrm{~d} 45 \mathrm{~b} 1 \mathrm{fb} 0 \mathrm{~b} \&$ version $=-1$.
20 National Institute for Health and Clinical Excellence. Specialist neonatal care quality standard. Quality Standards Programme, 2011. www.nice.org.uk/guidance/ qualitystandards/specialistneonatalcare/specialistneonatalcarequalitystandard.jsp.

21 Kirrane $H$. The chance of a lifetime? Bliss baby report 2010. Bliss, 2010.

22 Honest H, Forbes CA, Duree KH, Norman G, Duffy SB, Tsourapas A, et al. Screening to prevent spontaneous preterm birth: systematic reviews of accuracy and effectiveness literature with economic modelling. Health Technol Assess 2009;13:1-627.

23 Chung JH, Phibbs CS, Boscardin WJ, Kominski GF, Ortega AN, Gregory KD, et al. Examining the effect of hospital-level factors on mortality of very low birth weight infants using multilevel modeling. J Perinatol 2011;31:770-5.

24 Santhakumaran S, Statnikov Y, Manktelow B, Nagarajan S, Modi N, on behalf of the Neonatal Data Analysis Unit. Neonatal specialist care in England: report on mortality in 2009. Imperial College London, 2010.

25 Chien LY, Whyte R, Aziz K, Thiessen P, Matthew D, Lee SK. Improved outcome of preterm infants when delivered in tertiary care centers. Obstet Gynecol 2001;98:247-52.

26 Lui K, Abdel-Latif ME, Allgood CL, Bajuk B, Oei J, Berry A, et al. Improved outcomes of extremely premature outborn infants: effects of strategic changes in perinatal and retrieval services. Pediatrics 2006;118:2076-83.

27 Zeitlin J, Ancel PY, Delmas D, Breart G, Papiernik E. Changes in care and outcome of very preterm babies in the Parisian region between 1998 and 2003. Arch Dis Child Fetal Neonatal Ed 2010;95:F188-93.

28 Binder S, Hill K, Meinzen-Derr J, Greenberg JM, Narendran V. Increasing VLBW deliveries at subspecialty perinatal centers via perinatal outreach. Pediatrics 2011;127:487-93.

29 Phibbs CS, Baker LC, Caughey AB, Danielsen B, Schmitt SK, Phibbs RH. Level and volume of neonatal intensive care and mortality in very-low-birth-weight infants. N Engl J Med 2007;356:2165-75.

30 Scottish Executive. Introduction of managed clinical networks within the NHS in Scotland. NHS Management Executive, 1999. www.sehd.scot.nhs.uk/mels/1999_10.htm.

31 Guthrie B, Davies H, Greig G, Rushmer R, Walter I, Duguid A, et al. Delivering health care through managed clinical networks (MCNs): lessons from the North. National Institute for Health Research Service Deilvery and Organisation programme. Stationery Office, 2010. www.sdo.nihr.ac.uk/files/project/103-final-report.pdf.

32 Provan KG, Fish A, Sydow J. Interorganizational networks at the network level: a review of the empirical literature on whole networks. J Management 2007;33:479-516.

33 Goodwin N, Perri 6, Peck E, Freeman T, Posaner R. Managing across diverse networks of care: lessons from other sectors. Report to the National Co-ordinating Centre for NHS Service Delivery and Organisation R\&D. 2004. www.sdo.nihr.ac.uk/files/adhoc/39-policyreport.pdf.

34 Greene A, Pagliari C, Cunningham S, Donnan P, Evans J, Emslie-Smith A, et al. Do managed clinical networks improve quality of diabetes care? Evidence from a retrospective mixed methods evaluation. Qual Saf Health Care 2009;18:456-61.

\section{Accepted: 07 February 2012}

\section{Cite this as: BMJ 2012;344:e2105}

This is an open-access article distributed under the terms of the Creative Commons Attribution Non-commercial License, which permits use, distribution, and reproduction in any medium, provided the original work is properly cited, the use is non commercial and is otherwise in compliance with the license. See: http://creativecommons.org/licenses/bync/2.0/ and http://creativecommons.org/licenses/by-nc/2.0/legalcode. 


\section{Tables}

Table 1| Population characteristics of babies born at 27-28 weeks' gestation in epoch one (September 1998-August 2000, data from England, Wales, and Northern Ireland) and epoch two (January 2009-December 2010, data from England only). Figures are numbers (percentages) of babies

\begin{tabular}{|c|c|c|}
\hline & Epoch 1 & Epoch 2 \\
\hline \multicolumn{3}{|l|}{ Sex* } \\
\hline Female & $1592(45)$ & $1333(46)$ \\
\hline Male & $1930(55)$ & $1584(54)$ \\
\hline Missing & 0 & 2 \\
\hline Total & 3522 & 2919 \\
\hline \multicolumn{3}{|l|}{ Plurality† } \\
\hline Singletons & $2675(76)$ & $2134(73)$ \\
\hline Twins & $752(21)$ & $700(24)$ \\
\hline Triplets/quads & $95(3)$ & $85(3)$ \\
\hline Missing & 0 & 0 \\
\hline Total & 3522 & 2919 \\
\hline \multicolumn{3}{|c|}{ Birth weight (g)‡ } \\
\hline$\leq 500$ & $35(1)$ & $16(1)$ \\
\hline $501-750$ & 389 (11) & $239(8)$ \\
\hline $751-1000$ & $1072(30)$ & $884(30)$ \\
\hline $1001-1250$ & $1447(41)$ & $1299(45)$ \\
\hline $1251-1500$ & $502(14)$ & $451(16)$ \\
\hline$\geq 1501$ & $72(2)$ & $30(1)$ \\
\hline Missing & 5 & 0 \\
\hline Total & 3522 & 2919 \\
\hline
\end{tabular}

*From $\chi^{2}$ test $P=0.69$ for difference between epochs (missing values excluded). †From $\chi^{2}$ test $P=0.03$ for difference between epochs (missing values excluded). $\ddagger$ From $X^{2}$ test $P<0.001$ for difference between epochs (missing values excluded). 
Table 2| Number of babies born at 27-28 weeks' gestation in epoch one (September 1998-August 2000, data from England, Wales, and Northern Ireland) and epoch two (January 2009-December 2010, data from England only) by hospital of birth (categorised by annual number of neonatal intensive care days provided ${ }^{\star}$ ). Figures are numbers of babies (percentage of total) $\dagger$

\begin{tabular}{|c|c|c|}
\hline Days & Epoch 1 & Epoch 2 \\
\hline None & $139(4)$ & 0 \\
\hline $1-499$ & $843(24)$ & 331 (12) \\
\hline $500-999$ & $673(19)$ & $465(17)$ \\
\hline $1000-1499$ & $653(19)$ & $316(12)$ \\
\hline 1500-1999 & $556(16)$ & $287(11)$ \\
\hline$\geq 2000$ & $631(18)$ & $1325(49)$ \\
\hline Missing & 27 & 195 \\
\hline Total & 3522 & 2919 \\
\hline
\end{tabular}

*See report of working group of the British Association of Perinatal Medicine and Neonatal Nurses Association on categories of babies requiring neonatal care. ${ }^{11}$ †From $X^{2}$ test $P<0.001$ for difference between epochs (missing values excluded). 
Table 3| Number of babies born at 27-28 weeks' gestation in epochs 1 (September 1998-August 2000) and 2 (January 2009-December 2010), in England only, by transfer status. Figures are numbers of babies (percentage of total)*

\begin{tabular}{lcc} 
Transfer status & Epoch 1 & Epoch 2 \\
Not transferred & $2434(75)$ & $1919(66)$ \\
\hline Transferred $\leq 24$ hours & $235(7)$ & $360(12)$ \\
\hline Transferred 24 hours-28 days & $579(18)$ & $640(22)$ \\
\hline Missing & 0 & 0 \\
\hline Total & 3248 & 2919 \\
\hline
\end{tabular}

*From $\chi^{2}$ test $P<0.001$ for difference between epochs (missing values excluded). 
Table 4| Survival at 28 days of babies born at 27-28 weeks' gestation by epoch (epoch one September 1998-August 2000; epoch two January 2009-December 2010) in England only. Figures are numbers of babies (percentage of total)*

\begin{tabular}{lcc} 
& Epoch 1† & Epoch 2‡ \\
Survived & $2859(88)$ & $2677(94)$ \\
\hline Died & $389(12)$ & $182(6)$ \\
\hline Missing & 0 & 60 \\
\hline Total & 3248 & 2919 \\
\hline
\end{tabular}

${ }^{*}$ From $\chi^{2}$ test $\mathrm{P}<0.001$ for difference between epochs (missing values excluded). †Denominator=all live births.

‡Denominator=babies admitted to neonatal unit. 
Table 5| Number of babies born at 27-28 weeks' gestation by transfer status and hospital of birth (categorised by annual number of intensive care days provided in epoch two (January 2009-December 2010). ${ }^{*}$ Figures are numbers (percentages) of babies

\begin{tabular}{|c|c|c|c|c|c|c|}
\hline & \multicolumn{6}{|c|}{ Intensive care days provided in hospital of birth } \\
\hline & $1-499$ & $500-999$ & 1000-1499 & 1500-1999 & $>\mathbf{2 0 0 0}$ & Missing \\
\hline Not transferred & $142(42)$ & $329(71)$ & $238(75)$ & $221(77)$ & $925(70)$ & 64 \\
\hline Transferred $\leq 24$ hours & $153(46)$ & $57(12)$ & $17(5)$ & $9(3)$ & $88(7)$ & 36 \\
\hline $\begin{array}{l}\text { Transferred } 24 \text { hours-28 } \\
\text { days }\end{array}$ & $36(11)$ & $79(17)$ & $61(19)$ & $57(20)$ & $312(24)$ & 95 \\
\hline Missing & 0 & 0 & 0 & 0 & 0 & 0 \\
\hline Total & $331(100)$ & $465(100)$ & $316(100)$ & $287(100)$ & $1325(100)$ & 195 \\
\hline
\end{tabular}

*See report of working group of the British Association of Perinatal Medicine and Neonatal Nurses Association on categories of babies requiring neonatal care. ${ }^{11}$ 
Table 6 | Number of neonatal units by designation ${ }^{\star}$ in relation to annual number of neonatal intensive care days $\nmid$ provided and annual number of very low birthweight ( $<1500 \mathrm{~g}$ ) babies admitted in epoch two (January 2009-December 2010)

\begin{tabular}{|c|c|c|c|c|}
\hline & \multicolumn{3}{|c|}{ No of neonatal units } & \multirow[b]{2}{*}{ No of neonatal units (\% of total) } \\
\hline & Level 1 & Level 2 & Level 3 & \\
\hline \multicolumn{5}{|c|}{ Neonatal intensive care days: } \\
\hline$\leq 499$ & 32 & 23 & 0 & $55(38)$ \\
\hline $500-999$ & 2 & 29 & 1 & $32(22)$ \\
\hline $1000-1499$ & 0 & 12 & 4 & $16(11)$ \\
\hline $1500-1999$ & 0 & 5 & 6 & $11(8)$ \\
\hline$\geq 2000$ & 0 & 3 & 29 & $32(22)$ \\
\hline Total & 34 & 72 & 40 & $146(100)$ \\
\hline \multicolumn{5}{|c|}{ Very low birthweight babies admitted: } \\
\hline$\leq 24$ & 30 & 21 & 1 & $52(36)$ \\
\hline $25-49$ & 4 & 38 & 1 & $43(29)$ \\
\hline $50-99$ & 0 & 13 & 27 & $40(27)$ \\
\hline$\geq 100$ & 0 & 0 & 11 & $11(8)$ \\
\hline Total & 34 & 72 & 40 & $146(100)$ \\
\hline
\end{tabular}

${ }^{*}$ See BAPM report. ${ }^{14}$

†See report of working group of the British Association of Perinatal Medicine and Neonatal Nurses Association on categories of babies requiring neonatal care. ${ }^{11}$ 
Table 7 | Number of neonatal units shown in relation to annual number of very low birthweight $(<1500 \mathrm{~g})$ babies admitted and number of intensive care days provided in epoch two* (January 2009-December 2010)

\begin{tabular}{lccccc} 
& \multicolumn{2}{c}{ No of neonatal units } & No of neonatal units (\% of \\
total)
\end{tabular}

*See report of working group of the British Association of Perinatal Medicine and Neonatal Nurses Association on categories of babies requiring neonatal care. ${ }^{11}$ 